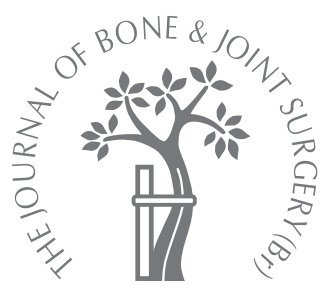

\title{
A correlation between the timing of biopsy after autologous chondrocyte implantation and the histological appearance
}

\author{
P. D. Gikas, \\ T. Morris, \\ R. Carrington, \\ J. Skinner, \\ G. Bentley, \\ T. Briggs \\ From the Royal \\ National \\ Orthopaedic \\ Hospital, Stanmore, \\ England
}

ㅍ. P. D. Gikas, MRCS, Academic Clinical Fellow

R. Carrington, FRCS(Orth), Consultant Orthopaedic

Surgeon

J. Skinner, FRCS(Orth),

Consultant Orthopaedic

Surgeon

G. Bentley, DSc, ChM, FRCS,

FMedSci, Professor

- T. Briggs, $\mathrm{MCh}$ (Orth)

FRCS(Orth), Consultant

Orthopaedic Surgeon, Medical

Director

Joint Reconstruction Unit

T. Morris, BSc, Clinical

Statistician

MRC Clinical Trials Unit

The Royal National

Orthopaedic Hospital, Brockley

Hill, Stanmore, Middlesex HA7

4LP, UK

Correspondence should be sent to Mr P. D. Gikas; e-mail: pdgikas@doctors.org.uk

(C)2009 British Editorial Society of Bone and Joint Surgery doi:10.1302/0301-620X.91B9.

$22490 \$ 2.00$

$J$ Bone Joint Surg $[\mathrm{Br}]$

2009;91-B:1172-7.

Received 19 February 2009;

Accepted 24 April 2009

\begin{abstract}
Autologous chondrocyte implantation is an option in the treatment of full-thickness chondral or osteochondral injuries which are symptomatic. The goal of surgery and rehabilitation is the replacement of damaged cartilage with hyaline or hyaline-like cartilage, producing improved levels of function and preventing early osteoarthritis. The intermediate results have been promising in terms of functional and clinical improvement.

Our aim was to explore the hypothesis that the histological quality of the repair tissue formed after autologous chondrocyte implantation improved with increasing time after implantation.

In all, 248 patients who had undergone autologous chondrocyte implantation had biopsies taken of the repair tissue which then underwent histological grading. Statistical analysis suggested that with doubling of the time after implantation the likelihood of a favourable histological outcome was increased by more than fourfold ( $p<0.001$ ).
\end{abstract}

Chondral damage to the knee is common in young patients. ${ }^{1,2}$ In symptomatic patients the current surgical treatment has focused on filling the defect with fibrocartilage from the subchondral bone. However, this tissue is deficient in type-II collagen and has poor resistance to shear forces which often leads to failure and early degenerative osteoarthritis (OA). ${ }^{3,4}$

The success of autologous chondrocyte implantation (ACI), as an alternative treatment for symptomatic chondral defects, was first reported by Brittberg et al in 1994. ${ }^{5}$ The technique involves filling the defect with the patient's own cells to restore the hyaline cartilage. These cells are harvested from the margin of the trochlear groove of the patient, cultured in the laboratory and re-implanted beneath a periosteal membrane to contain the cells within the defect. The membrane is sutured over the defect and sealed with fibrin glue to ensure that it remains watertight. In their study Brittberg et $\mathrm{al}^{5}$ demonstrated a hyaline-type repair, with the presence of typeII collagen, at one year in 11 of 15 patients with femoral condylar grafts. Further reports have suggested that the regenerate is durable with continued symptomatic improvement up to 11 years. ${ }^{6-16}$ There remains concern about the quality of the cartilage formed at the site of the defect. Needle biopsies taken after surgery have shown that this tissue is often fibrocartilage. ${ }^{7}$
Our aim was to explore the hypothesis that the quality of the repair tissue formed after ACI improves with time after surgery.

\section{Patients and Methods}

Clinical study. This prospective study was carried out in one centre. Ethical approval had been obtained. Since 1998, all patients undergoing ACI or matrix-assisted chondrocyte implantation (MACI) have been entered into the study. A periosteal flap technique was the only method available until 2003 when MACI was developed. Since then patients have been randomised either to ACI-C (collagen-covered ACI) or MACI. In all, 248 patients (136 males, 112 females) with a mean age of 33.4 years ( 15 to 52 ) have been operated on. The primary indication for surgery was persistent pain resulting from an isolated osteochondral defect greater than $1 \mathrm{~cm}^{2}$ in the articular surface of the knee. The mean follow-up was 32 months (12 months to 9 years). A total of 102 patients underwent ACI and 146 patients had MACI.

Trauma, osteochondritis dissecans and chondromalacia patellae were the principal indications for surgery with a large proportion of patients having previously undergone other surgical treatments. Instability of the joint, abnormal alignment, OA and inflammatory joint disease were all exclusion criteria. All patients entered a structured rehabilitation programme. 


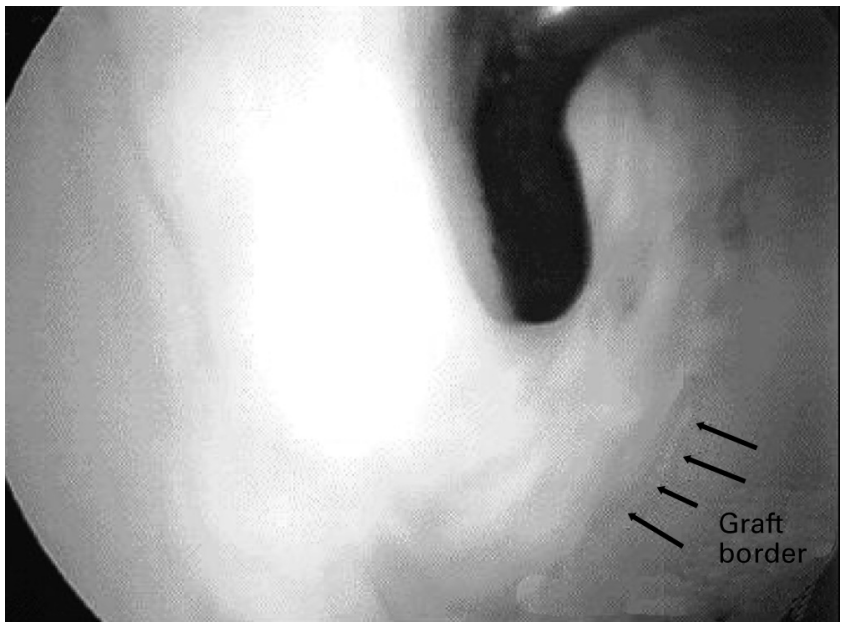

Fig. 1a

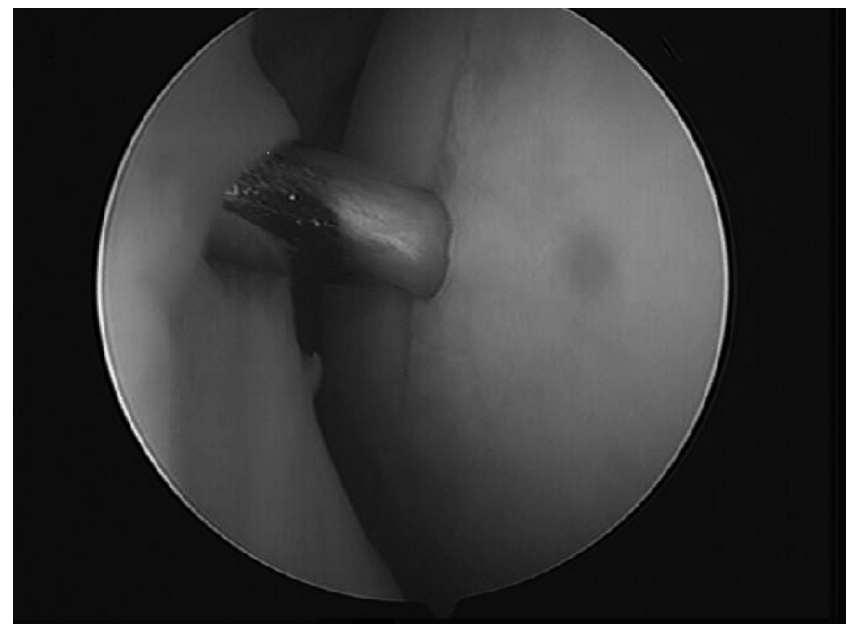

Fig. 1b

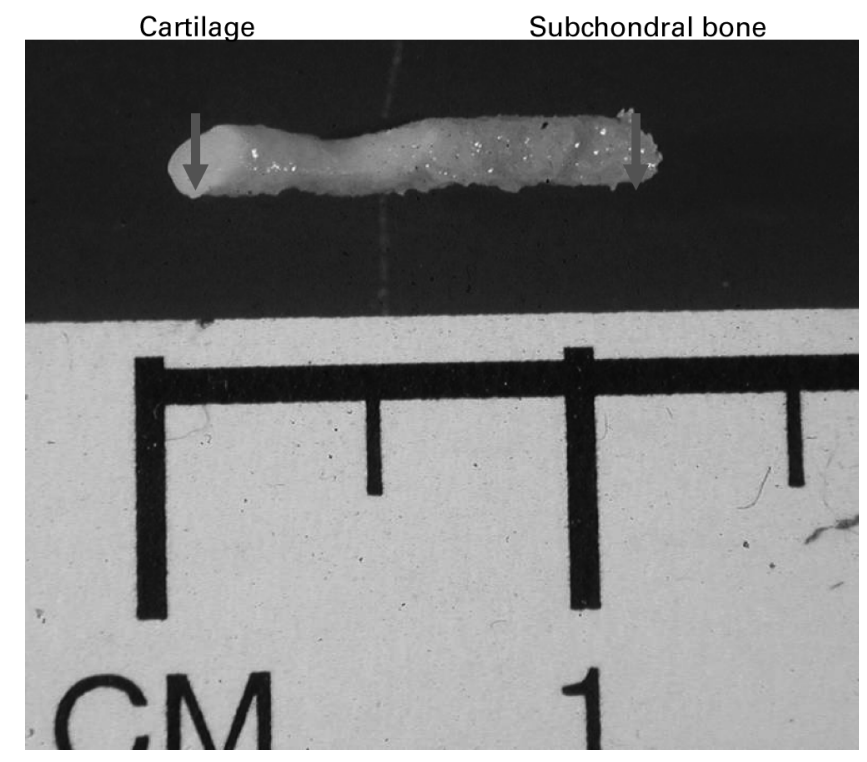

Fig. 1c

Demonstration of the biopsy technique from the centre of the resurfaced lesion using a Jamshidi needle at follow-up at one year showing a) the repaired defect, b) the needle inserted into the repair tissue and c) the biopsy core.

In addition to the normal pre-operative investigations, the patients were assessed before chondrocyte implantation using validated clinical scoring systems such as the modified Cincinnati rating score ${ }^{17}$ (0 to 100$)$, the Bentley functional rating score ${ }^{18}(0$ to 4$)$ and the Brittberg score ${ }^{5}$ (poor, fair, good or excellent). The annual functional outcome was assessed both at outpatient review and using postal questionnaires of the same scoring systems. The response rate was $99 \%$ throughout all the assessments with only two patients being lost to follow-up.

According to the study protocol, biopsies were scheduled for one year after implantation.

Biopsy procedure. At arthroscopy full-thickness biopsies were taken from the centre of the resurfaced lesion using a
Jamshidi needle with a diameter of $2 \mathrm{~mm}$ (Fig. 1). The biopsy core was processed and $5 \mu \mathrm{m}$ thick sections were stained with Erlich's haematoxylin and eosin and Safranin O (Fig. 2). Slides were subsequently examined microscopically under polarised light, and assigned to one of four categories as follows: fibrous tissue, fibrocartilage, mixed fibrohyaline and hyaline-like cartilage. Because of constraints of the National Health System and waiting-list targets, the biopsies were actually performed at various time points after implantation, allowing us to correlate statistically the histological findings with the maturity of the repair tissue. In total, 248 biopsies were performed with the mean timing of the biopsy being 14.8 months (3 to 55) after implantation. 


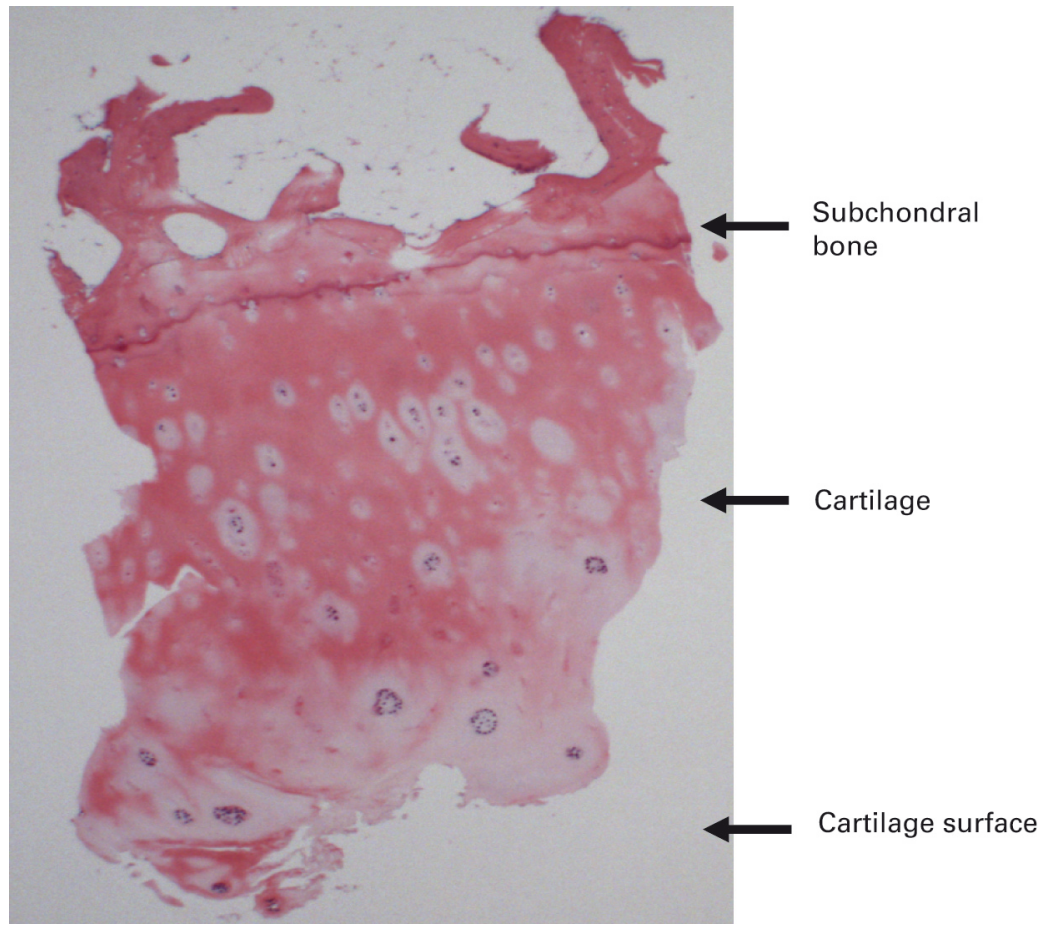

Fig. 2a

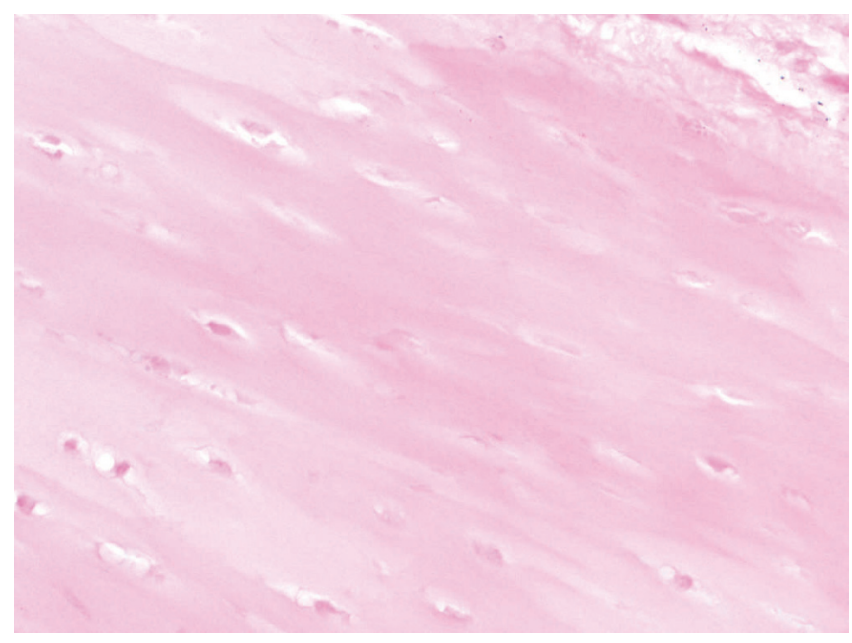

Fig. 2b

Photomicrographs of biopsies taken showing a) a hyaline-like repair tissue attached to subchondral bone with cells in the lacunae and a well-stained matrix (Safranin $O \times 10$ ) and b) cellular fibrocartilagenous repair tissue (haematoxylin and eosin $\times 20$ ).

Statistical analysis. Data from this study were based on the histopathological assessment of biopsies obtained after chondrocyte implantation according to the categories given above. These data were categorical and ordered so that ordinal regression could be used to make inferences. Patients with fibrous tissue histology, histologically very similar to fibrocartilage, were grouped with fibrocartilage for modelling purposes, giving three categories.

Various ordinal regression models were fitted to the data. A continuation ratio model showed significant improvement to a proportional odds model, but the full multinomial model, ignoring ordering, did not produce markedly superior fitting. Therefore, we used the continuation ratio model.

Biopsy times followed a skewed distribution and therefore a log transformation of the variable was performed to help to stabilise the variance. Even after this transformation the variable did not seem to follow a normal distribution so that non-parametric bootstrap sampling was used to obtain appropriate confidence intervals using the percentile method. 


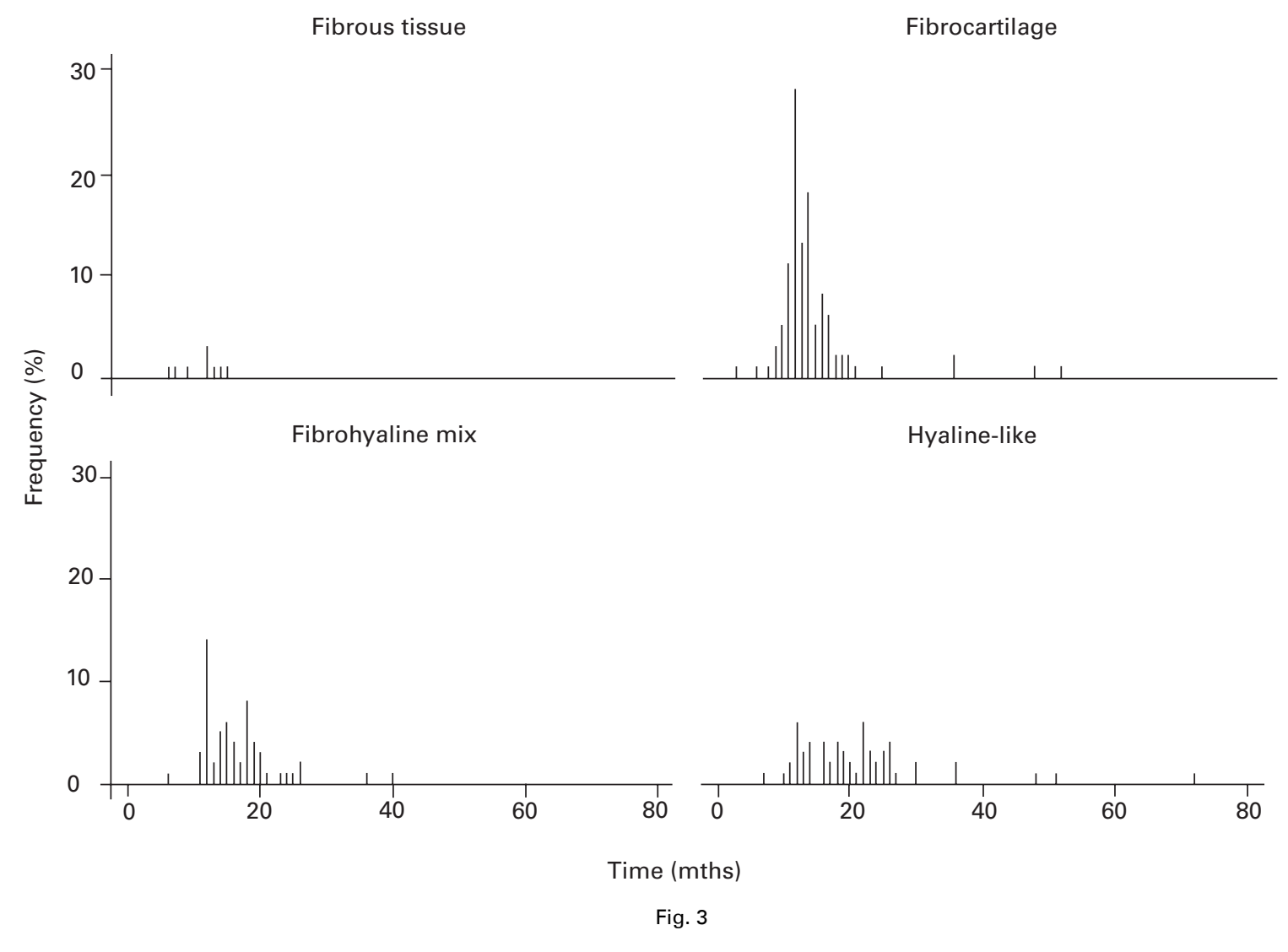

Spikeplots of the data according to the histological result. The distribution of the biopsy times is skewed.

A likelihood ratio test was performed across parameters to test whether the model parameters were constant across the categories of histology. Lack of fit was tested using a Pearson chi-squared test. Expected values were predicted from parameters of the continuation ratio model. Since time was measured on a continuous scale, the predicted value and times were calculated and then both observed and expected categorised to 0 to 12,13 to 18 and 18 months. Treatment differences between changes in the clinical scores were analysed using the unpaired $t$-test. Statistical significance was set at a p-value $\leq 0.05$.

\section{Results}

There was a sequential annual statistically significant improvement in the mean modified Cincinatti rating score for ACI and MACI $(\mathrm{p}<0.001)$ when compared with the pre-operative level. ${ }^{19}$

There was no significant difference $(\mathrm{p}>0.05)$ in the mean Bentley functional rating score between ACI and MACI up to two years after surgery, although both techniques showed significant and sequential improvement $(\mathrm{p}<0.001)$ when compared with their mean pre-operative score. ${ }^{19}$

The Brittberg score allowed patients to score knee function subjectively as poor, fair, good or excellent. We have recently published figures ${ }^{19}$ showing the sequential results for both ACI and MACI with responses being grouped as excellent or good compared with fair or poor. Continued sequential increases in beneficial responses (good or excellent) were observed for both techniques, compared with those obtained pre-operatively while there was a corresponding reduction in adverse responses. The rate of improvement of beneficial responses and the rate of decline of adverse responses, using the trendline gradient, was three times greater for MACI than for ACI.

In all, 59 biopsies showed hyaline tissue $(23.8 \%)$, 67 mixed hyaline and fibrocartilage $(27.0 \%), 113$ fibrocartilage only $(45.6 \%)$ and nine fibrous tissue $(3.6 \%)$. Figure 3 shows spikeplots of the data according to the histological grading. The distribution of the biopsy times is skewed.

Figure 4 shows a scatter plot of the same data along with a locally weighted scatterplot smoother. The latter uses locally weighted least-squares regressions to give an approximate smoothing idea of the relationship between time and histological outcome. A steep increase (improvement) in histological grade over time was observed.

In order to quantify this relationship and its strength, a continuation ratio model was used regressing histology on the natural logarithm of time. The odds ratio, $4.21(95 \%$ bootstrap confidence interval (CI) 2.56 to $8.41, \mathrm{p}<0.001$ ), estimates odds of two types of histology, the mixed fibrohyaline and hyaline-like cartilage compared with fibrous tissue and fibrocartilage, and simultaneously the odds of 


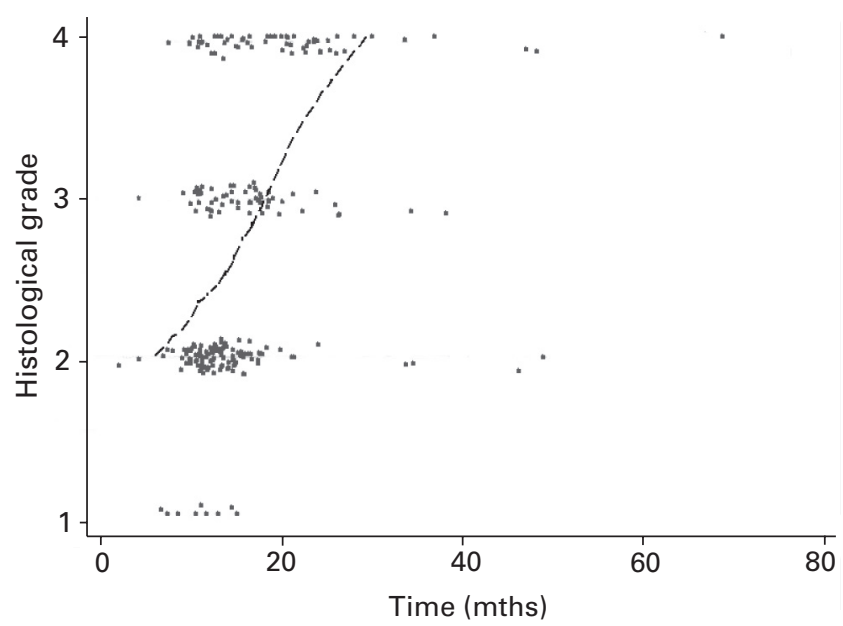

Fig. 4

Scatterplot showing the histological grading to time with a steep increase in improved histological grade with time.

hyaline-like histology compared with all fibrohyaline cartilage. In other words, if the time after implantation doubles it is 4.21 times more likely that the histological result will be consistent with hyaline-like tissue as opposed to any other histological outcome. This is a very strong dependency, and suggests that there is no purpose in taking biopsies before a certain time, given that the graft may still be improving.

A test for the equality of parameters across histological categories gave a p-value of 0.24 , indicating that one global odds ratio was sufficient. The Pearson test for lack of fit returned a p-value of 0.16 , again indicating there was no significant lack of fit from the model.

\section{Discussion}

After ACI, maturation of the cartilage occurs through several phases. ${ }^{3,11,20}$ The first, termed the proliferative phase, occurs during the first six weeks. Cells adhere to the subchondral bone plate, a process that can take from 12 to 18 hours. A soft primitive repair tissue forms during this initial phase. The second phase, the transition phase, occurs during the next four to six months. This is characterised by expansion of the matrix released by the chondrocytes with a putty-like consistency. The final phase, the matrix-remodelling phase, is characterised by progressive hardening of the cartilage tissue to the firm quality of the adjacent native cartilage. This process begins at approximately six months.

In vitro studies so far have shown that tissue-engineered autologous cartilaginous tissue attached to a bone substitute in vitro matures with time showing improved histomorphometric characteristics and integration. ${ }^{21}$ Recently, Brun et a ${ }^{22}$ have studied the postimplantation biopsies of 63 patients treated by grafts of autologous chondrocytes grown on three-dimensional hyaluronic-acid biomaterials. They found that the percentage of hyaline-regenerated tissue was significantly greater in biopsies obtained after, as opposed to within, 18 months of implantation.

Our results showed that compared with the preoperative scores, significant, continuous improvement was reported in all three functional scores $(\mathrm{p}<0.001)$ with annual maintained improvement up to nine years after implantation. Importantly, our results confirm our initial hypothesis that ACI forms a durable repair tissue which remodels and continues to improve in quality with time.

On the basis of our study it is proposed that for future research purposes 24 months should be used as the optimal time for performing a biopsy, since our data demonstrate that the histological outcome is still improving at this point.

No benefits in any form have been received or will be received from a commercial party related directly or indirectly to the subject of this article.

\section{References}

1. Bentley G. Robert Jones Lecture. Royal College of Surgeons of England. Presented at British Orthopaedic Association Annual Meeting 2007.

2. Curl WW, Krome J, Gordon ES, et al. Cartilage injuries: a review of 31,516 knee arthroscopies. Arthroscopy 1997;13:456-60.

3. Jones DG, Peterson L. Autologous chondrocyte implantation. J Bone Joint Surg [Am] 2006;88-A:2502-20.

4. Mankin HJ. The response of articular cartilage to mechanical injury. J Bone Joint Surg [Am] 1982;64-A:460-6.

5. Brittberg M, Lindahl A, Nilsson A, et al. Treatment of deep cartilage defects in the knee with autologous chondrocyte transplantation. N Eng/ J Med 1994;331:889-95.

6. Minas T. Chondrocyte implantation in the repair of chondral lesions of the knee: economics and quality of life. Am J Orthop 1998;27:739-44.

7. Peterson L, Minas T, Brittberg M, et al. Two- to 9 -year outcome after autologous chondrocyte transplantation of the knee. Clin Orthop 2000;374:212-34.

8. Peterson L, Brittberg M, Kiviranta I, Akerlund EL, Lindahl A. Autologous chondrocyte transplantation: biomechanics and long-term durability. Am J Sports Med 2002;30:2-12.

9. Peterson L, Minas T, Brittberg M, Lindahl A. Treatment of osteochondritis dissecans of the knee with autologous chondrocytes transplantation. J Bone Joint Surg [Am]2003;85-A(Suppl 2):17-24

10. Bentley G, Biant LC, Carrington RW, et al. A prospective, randomised comparison of autologous chondrocyte implantation versus mosaicplasty for osteochondral defects in the knee. J Bone Joint Surg [Br] 2003;85-B:223-30.

11. Henderson I, Francisco R, Oakes B, Cameron J. Autologous chondrocytes implantation for treatment of chondral defects of the knee: a clinical, arthroscopic, MRI and histologic evaluation at 2 years. Knee 2005;12:209-16.

12. Peterson L. Articular cartilage injuries treated with autologous chondrocytes implantation in the human knee. Acta Orthop Belg 1996;62(Suppl 1):196-200.

13. Micheli LJ, Browne JE, Erggelet C, et al. Autologous chondrocytes implantation of the knee: multicentre experience and minimum 3 year follow up. Clin J Sports Med 2001;11:223-8

14. Knutsen G, Engebretsen L, Ludvigsen TC, et al. Autologous chondrocytes implantation compared with microfracture in the knee: a randomised trial. J Bone Joint Surg [Am]2004;86-A:455-64.

15. Mithofer K, Peterson L, Mandelbaum BR, Minas T. Articular cartilage repair in soccer players with autologous chondrocytes transplantation: functional outcome and return to competition. Am J Sports Med 2005;33:1639-46.

16. Browne JE, Anderson AF, Arciero R. Clinical outcome of autologous chondrocytes implantation at 5 years in US subjects. Clin Orthop 2005;436:237-45.

17. Noyes FR, Mooar LA, Barber SD. The assessment of work-related activities and limitations in knee disorders. Am J Sports Med 1991;19:178-8. 
18. Meister K, Cobb A, Bentley G. Treatment of painful articular cartilage defects of the patella by carbon-fibre implants. J Bone Joint Surg [Br] 1998;80-B:965-70.

19. Gikas PD, Bayliss L, Bentley G, Briggs TWR. Overview of autologous chondrocyte implantation. J Bone Joint Surg [Br] 2009;91-B:997-1006.

20. Hollander AP, Dickinson SC, Sims TJ, et al. Maturation of tissue engineered cartilage implanted in injured and osteoarthritic human knees. Tissue Engineering 2006;12:1787-98
21. Petersen JP, Ueblacker $\mathbf{P}$, Goepfert C, et al. Long term results after implantation of tissue engineered cartilage for the treatment of osteochondral lesions in a minipig model. J Mater Sci Mater Med 2008;19:2029-38.

22. Brun P, Dickinson SC, Zavan B, et al. Characteristics of repair tissue in secondlook and third-look biopsies from patients treated with engineered cartilage: relationship to symptomatology and time after implantation. Arthritis Res Ther 2008;10:132. 